Journal of Emergency Primary Health Care

An International elournal of Prehospital Care Research, Education, clinical Practice, Policy and Service Delivery

ISSN 1447-4999

\title{
CLINICAL PRACTICE
}

Article 990179

\section{Social and environmental influences affecting the risk of development of meningococcal disease: considerations for prehospital care.}

\author{
Stephen Burgess \\ Lecturer, Centre for Ambulance and Paramedic Studies, Monash University, Melbourne
}

\begin{abstract}
After briefly reviewing the clinical features, major sequelae and initial treatment of invasive meningococcal disease, and reviewing the international and domestic incidence of meningococcal disease, the epidemiological risk factors for meningococcal disease found in the literature are summarised. These findings and the heterogeneity of sub-population risk, especially in relation to indigenous populations, are then discussed. The implications of these discussions are then applied to paramedic practice as a component of primary health care. The author argues that consideration of such data is important in developing recommendations for the development of system planning and clinical practice guidelines.
\end{abstract}

Keywords

clinical practice guidelines; meningococcal disease; prehospital care; risk factors; social influences.

\section{Introduction}

Meningococcal disease is not a common illness, but when it does occur it can cause a serious, potentially life threatening acute illness. ${ }^{1,2}$ After briefly examining meningococcal disease and the clinical syndromes that can follow infection, this paper will summarise the literature on the known risk factors and social influences that contribute to the risk of developing meningococcal disease. It will be argued that data about known risk factors and social influences on excess risk should be deliberately and explicitly considered when evaluating disease risk in various populations, and that such a process should play an important role in influencing the development of clinical practice guidelines for paramedics.

\section{Clinical features of meningococcal disease}

Meningococcal disease is a serious, potentially life threatening acute illness. It is a leading cause of bacterial meningitis and sepsis in children and young adults in Australia. ${ }^{3,4}$

At least four conditions have to be met before invasive disease can occur. These conditions are:

(i) exposure to a pathogenic strain

(ii) colonization of the naso-oropharyngeal mucosa

(iii) passage through that mucosa, and;

(iv) survival of the meningococcus in the bloodstream ${ }^{4}$. 
Meningococcal disease usually presents as meningitis or septicaemia, or a combination of the two ${ }^{5}$. Septicaemia can be particularly severe and has considerably greater mortality than meningococcal meningitis. Meningococcal septicaemia can have a fulminant and rapidly fatal course which causes meningococcal disease to be so feared by the general public, and may lead to diagnostic anxiety amongst clinicians. ${ }^{1}$

Because healthy young children are primarily the victims of this disease, its incidence continues to increase, and the mortality is still $10 \%$, social, medical and scientific vigilance is required to minimise the effects of disease. ${ }^{4}$ Acute meningococcal disease, in particular fulminant meningococcal sepsis, can be fatal within a few hours. Therefore, early diagnosis and immediate recognition of imminent deterioration is pivotal in managing fulminant meningococcal sepsis. Recognition of patients at risk is therefore crucial for the timely start of life-saving antibiotic and anti-shock therapy. ${ }^{4}$

\section{Sequelae in survivors}

Deafness is the single most common permanent deficit in survivors of meningococcal meningitis. It occurs in 4\%-6\% of survivors, in half of whom it is severe and bilateral ${ }^{6}$.

Deafness is more common in children than adults. Permanent motor deficits, retardation and hydrocephalus occur in less than $1 \%$ of survivors. ${ }^{6}$ A significant proportion of survivors will have tissue damage that requires surgical treatment, such as skin grafts, or partial or full amputation of limbs. ${ }^{1}$

While a detailed description of the various presentations and clinical features of meningococcal disease are beyond the scope of this paper, they have been very well discussed in detail elsewhere. ${ }^{4,7,8}$

It is clear from the clinical picture described above that meningococcal disease is a serious illness, where an appropriately high index of suspicion, early treatment and admission to a suitable hospital can have a major effect on both morbidity and mortality in the patient with meningococcal disease. Each of the components in successful diagnosis and treatment has implications for paramedics and those that develop the clinical practice guidelines (CPG's) that inform their practice.

\section{Epidemiology}

Meningococcal disease occurs worldwide as endemic infections, and less commonly as disease outbreaks. There are several main subgroups of $N$. meningitidis that can cause meningococcal disease. These are subgroups A, B, C, W135 and Y. ${ }^{7}$

Most cases of meningococcal disease are caused by serogroups $\mathrm{A}$ and $\mathrm{C}$, for which polysaccharide vaccines are effective, and serogroup B, which has a polysaccharide capsule that is poorly immunogenic in humans. ${ }^{7}$

The incidence of meningococcal disease during the last 30 years varied from 1-3/100,000 in most industrialized nations to $10-25 / 100,000$ in some third-world countries. ${ }^{4}$ These different attack rates reflect the different pathogenic properties of $N$. meningitidis strains and different socioeconomic, environmental, and climatic conditions. 


\begin{tabular}{|c|c|c|c|c|c|c|c|c|c|c|c|c|c|}
\hline \multicolumn{2}{|c|}{ B } & \multicolumn{2}{|c|}{ c } & \multicolumn{2}{|c|}{ A } & \multicolumn{2}{|c|}{$\mathrm{Y}$} & \multicolumn{2}{|c|}{ W135 } & \multicolumn{2}{|c|}{$\mathrm{NG}^{*}$} & \multicolumn{2}{|c|}{ TOTAL } \\
\hline $\mathrm{N}$ & $\%$ & $\mathrm{~N}$ & $\%$ & $\mathrm{~N}$ & $\%$ & $\mathrm{~N}$ & $\%$ & $\mathrm{~N}$ & $\%$ & $\mathrm{~N}$ & $\%$ & $\mathrm{~N}$ & $\%$ \\
\hline & 63 & 120 & 33 & 0 & 0 & 9 & 2.5 & 6 & 1.5 & 2 & 0.5 & 369 & 100 \\
\hline
\end{tabular}

Figure 1. N. meningitidis isolates, 1999 , by serogroups. ${ }^{*}$ NG $=$ non-groupable ${ }^{1}$

The overall incidence rate of meningococcal disease throughout the 1990s in Australia remained relatively stable ranging from 1.7 to 2.7 per 100,000 population, so approximately 600 cases occur nationally. ${ }^{1,2}$ Males develop meningococcal disease more often than females ${ }^{1}$. Throughout the 1990s there has been a bimodal distribution of the majority of notified cases with the highest rate in the 0-4 age group and a second peak in the 15-24 year age group, although all age groups can be affected, as demonstrated in Figure 2.

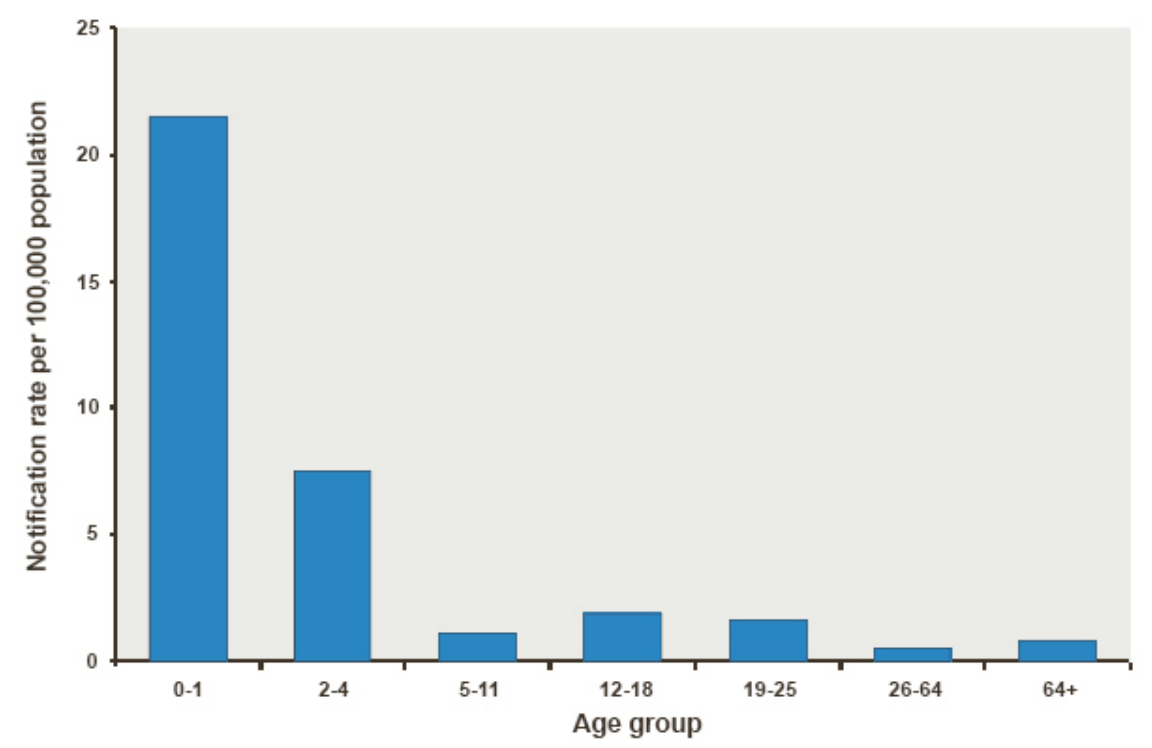

Figure 2. Age -specific notification rates of invasive meningococcal disease, Australia 1991-1999. ${ }^{1}$

Meningococcal disease characteristically has a seasonal pattern with a peak of incidence in the winter and spring months (see Figure 3 ). The reasons for this seasonality are not clear. ${ }^{1}$

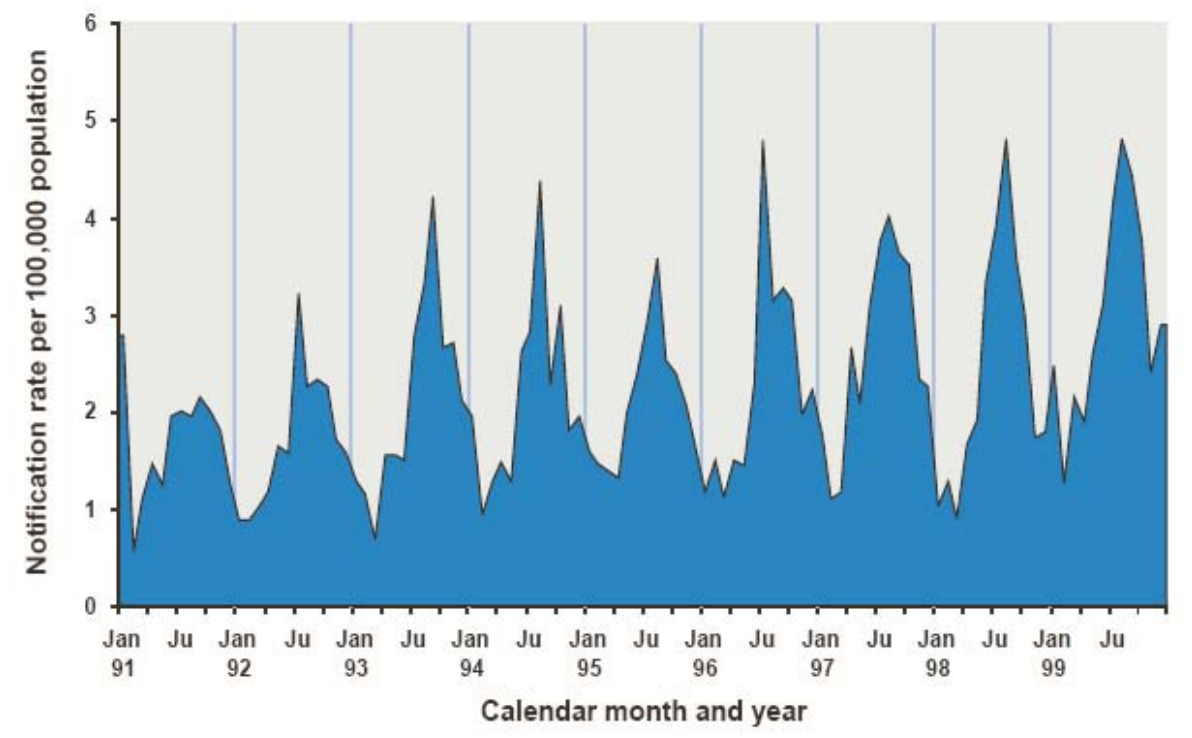

Figure 3. Notification rate of invasive meningococcal disease, Australia 1991-1999. ${ }^{1}$ 
In Australia, the overall notification rates per 100,000 in the 1990s ranged from a minimum of 1.7 per 100,000 in 1992 to a maximum of 2.7 per 100,000 in 1997. ${ }^{1}$ The highest State or Territory rate was 8.9 per 100,000 in the Northern Territory in 1998. Notification rates in other States ranged from 1.0-3.1 per 100,000 people. ${ }^{1}$ The high variant rate of disease notification in the Northern Territory is noteworthy, and the relative disease risk amongst indigenous populations will be discussed later in this paper.

Paramedics should bear age, gender and seasonal variations in mind when considering the likelihood of meningococcal disease in a particular case, particularly if provisional diagnosis is unclear or ambiguous. When integrated with the clinical presentations of disease mentioned earlier, an understanding of epidemiological trends in the disease can assist in early detection of likely cases.

\section{Risk factors for developing meningococcal disease}

The reasons why some people do get invasive meningococcal disease while most do not are poorly understood. It is likely that besides the factors related to the infectious agent or the host, environmental characteristics also play a role. ${ }^{9}$ A number of risk factors for the development of meningococcal disease have been identified. The epidemiological literature, although not extensive, is consistent. ${ }^{9}$ These risk factors can be broadly grouped into clinical versus environmental and social risks, and it is the latter that will be discussed here.

\section{Environmental and social risks}

Several recent studies have indicated that the risk of invasive meningococcal disease is influenced by environmental and social factors. ${ }^{2,3,9-28}$ Parental smoking appears to be a particularly strong risk factor for invasive meningococcal disease in children ${ }^{9}$. The frequency of the disease seems higher in low socio-economic areas, and all studies found that the risk of disease was lower in children living in more favourable socioeconomic conditions. ${ }^{11-30}$ The major factors for increased risk are: smoking, socio-economic disadvantage, dwelling crowding and poverty, and geographic risk.

\section{Smoking}

Several biological mechanisms can provide the link between tobacco smoke and meningococcal disease. ${ }^{9}$ Exposure to smoke causes direct damage to the nasopharyngeal mucosa, and passive smoking is associated with an increased risk of respiratory disease in young children ${ }^{9}$. As children have delicate mucous membranes, they may be more likely to acquire meningococcal disease if they are chronically exposed to passive smoking. ${ }^{9}$

Exposure to tobacco smoke has been consistently identified in case-control studies and the one reported cohort study of meningococcal disease. ${ }^{10}$ It is also known that smoking rates are higher in lower socioeconomic groups. ${ }^{22}$

In a case-control study investigating environmental factors in confirmed cases of meningococcal disease, passive smoking in the home (30 or more cigarettes daily) was associated with an odds ratio of 7.5 for disease risk in children aged under five. The odds ratios increased both with the numbers of cigarettes smoked and with the number of smokers in the household. ${ }^{25}$

\section{Socio economic disadvantage}

Existing data consistently suggests that socioeconomic disadvantage increases the risk of meningococcal disease. In Brazil, the incidence of meningococcal disease was about two times higher in low socio economic areas than in more affluent areas. ${ }^{9}$ In the UK, crowding and several other adverse social indicators were related to meningococcal disease risk. ${ }^{9,18}$ US 
studies have consistently found that people of African-American descent, people of low socioeconomic status, low maternal education and other adverse social characteristics were associated with increased risk of the disease. ${ }^{9}$ The risk of invasive meningococcal disease in children is strongly influenced by unfavourable socioeconomic circumstances ${ }^{9}$

\section{Dwelling crowding and poverty}

Across the international literature, dwelling crowding has been found to be associated with excess risk for the development of meningococcal disease. The authors of a longitudinal study of Belgian schoolchildren concluded that "populations of low socio-economic status and living in densely populated areas constitute a target population for meningococcal disease prevention". 15 (p.147)

A study of the descriptive epidemiology of a 10-year serogroups B meningococcal disea ase epidemic in New Zealand (1991-2000) found that overcrowded living conditions are contributing to the epidemic. ${ }^{10}$ This study also found that Maori age-standardised rates for meningococcal disease were 2.5 times and Pacific Islanders age-standardised rates were 5.5 times the rate of European New Zealanders.

Studies in Denmark found that the risk of invasive meningococcal disease increased with increasing household density, even after adjusting for confounders such as exposure to smoking, age and socio-economic factors. The authors suggested that the mechanism behind this association is "increased risk of exposure from carriers and more effective transmission". 16(p.20) Such a mechanism has also been proposed as a result of an Australian study. ${ }^{13}$ Similarly, another Australian study found that invasive meningococcal disease was associated with sharing bedrooms with two or more people. ${ }^{3}$ Dwelling crowding is strongly negatively correlated with almost all socio-economic indicators. As such it is an excellent indicator of both disadvantage and disease risk. ${ }^{13}$

These findings are of particular importance to paramedic practice. Unlike the majority of clinical interactions with other health professions, patient contact with paramedics frequently occurs in the patient's dwelling. In addition, a patient's clinical encounter with paramedics is almost never scheduled in advance, as may be the case with visiting nurses or general practitioners, for example. Paramedics then are uniquely placed to make assessments about crowding of dwelling conditions, exposure to tobacco smoke, and to gain an unvarnished (albeit necessarily subjective) impression of the patient's social and economic circumstances. These factors are known to strongly affect a person's risk for developing meningococcal disease. Astute clinicians will take these circumstances into account when assessing a patient with possible meningococcal disease, and when weighing the consequences of various treatment and transport options.

\section{Geographic risk}

A number of studies examining the geographic risk of developing meningococcal disease have been conducted. 2,10,14,18,20,26,28 Despite these studies being conducted in various countries, the results are consistent: the incidence of cases of meningococcal disease can be mapped accurately, and that when this is done cases overwhelmingly occur in lower socioeconomic areas. Given the known excess risk of ill health associated with socio-economic disadvantage, these findings may be unsurprising.

However well anticipated, these results carry important implications for health service planners, including managers in ambulance services. Planning service delivery and clinical practice at a macro level may be inadequate. The data suggest that where geographic data are overlayed with information about social-economic disadvantage, there is likely to be clear regions or areas within regions, which have excess risk of developing a particular disease e.g. Author(s): Stephen Burgess 
meningococcal disease. This suggests that a more sophisticated approach to planning and delivering health care is warranted. Where paramedics usually attend cases within a welldefined geographic area (as is the case in most parts of regional, rural and remote Australia), this planning may need to be done at the micro level - possibly branch by branch. Much of this information to enable these evaluations is already freely available from the Australian Bureau of Statistics at http://www.abs.gov.au.

\section{Heterogeneity of risk among specific sub-populations International data - Indigenous populations}

In the United States, people of African-American descent and persons of lower socioeconomic status (including indigenous populations) have consistently been found to be at higher risk for meningococcal disease than Caucasian and persons of higher socioeconomic status. ${ }^{7}$ Since 1990 New Zealand has been experiencing an epidemic of meningococcal disease. Age-standardised disease rates for Maori and Pacific Island people were three and six times higher respectively than for the European population. ${ }^{1}$

\section{Australian data - Indigenous populations}

Indigenous populations typically are at excess risk of disease. Among Native Americans and Australian Aboriginal groups in which there is a high incidence of SIDS, there is also an increased incidence of serious bacterial infections of the respiratory tract. ${ }^{29}$ It is accepted that in Australia, indigenous people are particularly at risk of developing meningococcal disease. The risk of sustained transmission of invasive meningococcal disease in indigenous communities, especially remote Aboriginal communities, is high. ${ }^{1}$

It is important for paramedics who attend such a case in an indigenous community, or who perform a subsequent patient transfer, to document the possibility of meningococcal disease and to make sure that this information is promptly passed on to local public health authorities to minimise the possibility of a local outbreak of disease.

Although not set in an indigenous population, a recent British ecological study confirmed the previously reported association between the incidence of meningococcal disease, and social deprivation in childhood, and shows for the first time showed an association between social deprivation and meningococcal mortality. Further, the authors make the following astute observation:

"Our findings suggest that although the main determinant of differences in disease mortality by area deprivation is risk of disease incidence, there may be an additional component due to socio-cultural differences in disease presentation and/or early management. Although minimisation of poverty will have the greatest impact on disease prevention, in the short to medium term, improvements in access to healthcare and earlier treatment are more likely to reduce the rate of mortality from meningococcal disease for all social groups". 20 (p.1067)

The challenge for all health service managers is this: improvement in treatment and better access to treatment, particularly for populations that are known to be isolated from good care by barriers such as geography, income, or cultural difference. 


\section{Discussion}

The epidemiological data and previous research have consistently demonstrated that the most disadvantaged areas have higher rates of meningococcal disease than the least disadvantaged areas, and the most deprived people within those areas, are at greatest risk of developing the disease. $^{26}$

The data clearly show that meningococcal disease incidence and mortality are socially patterned..$^{20}$ In Australia, Aboriginal and Torres Strait Islander people are, as a group, the most socially disadvantaged Australians by every standard measure. ${ }^{30}$ For all of the known risk factors for the development of invasive meningococcal disease, indigenous people are overwhelmingly over represented: poorer health status, recent illness, low socio-economic status, residing in a deprived area, smoking, exposure to tobacco smoke, dwelling crowding, low vaccination rates, access to and use of appropriate health services, as well as possible genetic influences. ${ }^{30}$ The implications of the data are unambiguous: indigenous populations in Australia and elsewhere are at excess risk for the development of meningococcal disease compared to the general population. ${ }^{27}$

When planning prehospital clinical treatment programs for communicable diseases, it should be obvious that that the risk of developing the disease of interest is often heterogeneous among sub-groups of the population. Yet, the development processes of CPG's for use by paramedics by the ambulance services in Australia $^{31-38}$ seem to repeatedly make the erroneous assumption of homogeneity of risk across populations. Nowhere in the Australian CPG's is there any mention of heterogeneous risk of specific disease/conditions (including meningococcal disease) which fall under their purview.

Such methodological shortcomings are not merely an historical artefact. For example, when documenting in detail the analysis that lead to the recent decision to not introduce benzyl penicillin for the treatment of meningococcal septicaemia into paramedic practice in Queensland ${ }^{39}$, the authors, due to the absence of explicit discussion of risk stratification, seem to assume homogeneity of disease risk across the population. Yet the risk of meningococcal disease amongst the population of Queensland is heterogeneous. Indigenous people have an excess risk of developing the disease compared to the general population of Queensland. Despite Queensland having a relatively large indigenous population, many of whom are culturally and geographically isolated from sophisticated health centres, no discussion of this population's known health needs and excess disease risk is canvassed.

While the absolute risk of developing meningococcal disease generally remains low, the relative risk of developing invasive meningococcal disease within specific (albeit small) populations, and the attributable risk which is associated, is large. This is a simple epidemiological concept, yet it consistently remains overlooked when decisions about paramedic clinical treatment programs are developed. These oversights have major implications for planning, diagnosis and treatment in paramedic practice.

The concepts and methodology canvassed in the pertinent paper by Australian geographic researchers Beggs and Siciliano, ${ }^{13}$ when discussing environmental risk for disease, capture this problem and lost opportunity well:

"Such oversights are unfortunate because evaluating the geographic distribution and environmental determinants of disease can result in many valuable insights, providing valuable information for public health authorities, for example in planning and funding of public health facilities. In addition, the identification of environmental determinants can result in recommendations for enhancing the management or prevention of disease. ${ }^{\text {13 (p.378) }}$ 
The association between areas of deprivation (particularly indigenous communities) and meningococcal disease is potentially of significant public health importance as it provides an avenue for population based primary prevention and intervention. ${ }^{20}$ The identification of preventable risk factors for invasive meningococcal disease is still of public health importance, ${ }^{26}$ and the identification of these needs to be considered by paramedics.

\section{Conclusion}

It is accepted that many infectious diseases preferentially affect the most disadvantaged in society. This has been discussed at global, national and even local levels. But epidemiological data and geographic studies have shown that it is holds true even when comparing small areas such as postal or electoral districts. The environmental and social factors conferred upon an individual or population through location, type of residence in a particular area, and the known risks of serious clinical disease to which they are exposed, may be as important as the individual 'risk factors' where communicable disease is concerned.

The relevant literature is not always to be found within refereed journals to which paramedics and emergency clinicians usually turn for information about clinical treatment. Refereed literature from other disciplines and the readily available geographic and demographic data need to be explicitly and conscientiously considered within a public health framework when formulating decisions about disease risk, clinical treatment, system planning and service delivery. Paramedic practice is sorely in need of a re-orientation of the way information is considered, and what data are considered. 


\section{References:}

1. Communicable Diseases Network Australia Guidelines for the early clinical and public health management of meningococcal disease in Australia, June 2001. In. ACT: Commonwealth Department of Health and Aged Care.; 2001.

2. Robinson P, Taylor K, T. N. Risk-factors for meningococcal disease in Victoria, Australia, in 1997. Epidemiol Infect. 2001;127(2):261-8.

3. McCall BJ, Neill AS, Young M M. Risk factors for invasive meningococcal disease in southern Queensland, 2000-2001. Internal Medicine Journal 2004;34(8):464-468.

4. van Deuren M, Brandtzaeg P, van der Meer JWM. Update on Meningococcal Disease with Emphasis on Pathogenesis and Clinical Management. Clin Microbiol Rev. 2000;13(1):144166.

5. Cooke ME. Prehospital Administration of Benzyl Penicillin in the UK. Journal of Emergency Primary Health Care 2005;3(1-2).

6. Grimwood K, Andersonb P, Andersonb V, Tanc L, Nolan T. Twelve year outcomes following bacterial meningitis: further evidence for persisting effects. Arch Dis Child 2000;83:111-116.

7. Rosenstein NE, Perkins BA, Stephens DS, Popovic T, Hughes JM. Medical Progress: Meningococcal Sisease. New England Journal of Medicine 2001;344(18):1378-1388.

8. Yung AP, McDonald MI. Early clinical clues to meningococcaemia. MJA. 2003(178):134137.

9. Kriz P, Bobak M, Kriz B. Parental smoking, socioeconomic factors, and risk of invasive meningococcal disease in children: a population based case-control study. Arch Dis Child 2000;83:117-121.

10. Baker MG, Martin DR, Kieft CEM, Lennon D. A 10-year serogroup B meningococcal disease epidemic in New Zealand: Descriptive epidemiology, 1991-2000. Journal of Paediatrics and Child Health Volume 37 Issue s5 Page 13 - October 2001 2001;37(s5):13.

11. Albrecht J, Ramasubramanian L. The Moving Target: A Geographic Index of Relative Wellbeing. Journal of Medical Systems 2004;28(4):371-384.

12. Blakel T, Atkinson J, Kiro C, Blaiklock A, D'Souza A Child mortality, socioeconomic position, and one-parent families: independent associations and variation by age and cause of death. International Journal of Epidemiology 2003;32:410-418.

13. Beggs PJ, Siciliano F. Spatial Relationship between Dwelling Crowding and Selected Causes of Morbidity in Sydney, Australia, 1994-97. Australian Geographer 2001;32(3):377-401.

14. Crump JA, Murdoch DR, Baker MG. Emerging Infectious Diseases in an Island Ecosystem: The New Zealand Perspective. Emerg Infectious Diseases. 2001;7(5):767-772.

15. De Wals P, Gilquin C, De Maeyer S, Bouckaert A, Noel A, Lechat MF, et al. Longitudinal study of asymptomatic meningococcal carriage in two Belgian populations of schoolchildren. J Infect. 1983;6(2):147-56.

16. Deutch S, Labouriau R, Schonheyeder HC, Ostergaard L, Norgard B, Sorensen HT. Crowding as a risk factor of meningococcal disease in Danish preschool children: a nationwide population-based case-control study. Scand J Infect Dis. 2004;36(1):20-3.

17. Dybing E, Sanner T. Passive smoking, sudden infant death syndrome (SIDS) and childhood infections. Hum Exp Toxicol. 1999;18(4):202-5.

18. Fone DL, Harries JM, Lester N, Nehaul L. Meningococcal disease and social deprivation: a small area geographical study in Gwent, UK. Epidemiol Infect. 2003;130(1):53-8.

19. Haralambous E, Weiss HA, Radalowicz A, Hibberd ML, Booy R, Levin M. Sibling familial risk ratio of meningococcal disease in UK caucasians. Epidemiology and Infection. 2003(130):413-418.

20. Heyderman RS, Ben-Shlomo Y, Brennan CA, Somerset M. The incidence and mortality for meningococcal disease associated with area deprivation: an ecological study of hospital episode statistics. Arch Dis Child. 2004;89(11):1064-8. 
21. Hoebe CJPA, de Melker H, Spanjaard L, Dankert J, Nagelkerke N. Space-Time Cluster Analysis of Invasive meningococcal Disease. Emerging Infectious Diseases 2004;10(9):1621-1626.

22. Iles K, Poplawsk NK, Coupe RTL. Passive exposure to tobacco smoke and bacterial meningitis in children. Journal of Paediatrics and Child Health. 2001;37(4):388.

23. Raghunathan PL, Bernhardt SA, NE. R. Opportunities for control of meningococcal disease in the United States. Annu Rev Med. 2004;55:333-53.

24. Ranta J, Makela PH, Takala A, Arjas E. Predicting the course of meningococcal disease outbreaks in closed subpopulations. Epidemiology and Infection 1999(123):359-371.

25. Stanwell-Smith RE et al. Smoking, the environment and meningococcal disease: a case control study. Epidemiology of Infections 1994;112:315-328.

26. Stuart JM, Middleton N, DJ G. Socioeconomic inequality and meningococcal disease. Commun Dis Public Health 2002;5(4):327-8.

27. Tobias M. Indicators of Inequality. Classification and selection of ethnic health disparity indicators. Wellington: Ministry of Health, Public Health Directorate; 2001 June.

28. Williams CJ, Willocks LJ, Lake IR, Hunter PR. Geographic correlation between deprivation and risk of meningococcal disease: an ecological study. BMC Public Health. 2004;4(1):30.

29. Blackwell CC, Weir DM, A B. Infection, inflammation and sudden infant death syndrome. Ambulatory Child Health 2000;6(s1):32.

30. Trewin D, Madden R. The Health and Welfare of Australia's Aboriginal and Torres Strait Islander Peoples. Canberra: Australian Institute of Health and Welfare; 2005.

31. ACT Ambulance Service. Clinical Management Manual. Canberra ACT: ACT Ambulance Service; 2004.

32. Ambulance Service of New South Wales. Protocols and Pharmacologies. Sydney: Ambulance service of New South Wales; 2005.

33. Metropolitan Ambulance Service and Rural Ambulance Victoria. Clinical Practice Guidelines. Melbourne: Metropolitan Ambulance Service and Rural Ambulance Victoria; 2005.

34. Queensland Ambulance Service. Queensland Ambulance Service Clinical Practice Manual. Brisbane: Queensland Ambulance Service; 2001.

35. South Australian Ambulance Service. Ambulance Officer Protocols. Adelaide: South Australian Ambulance Service; 2005.

36. St John Ambulance Service Australia, Western Australia Ambulance Service Inc. Clinical Practice Guidelines for Ambulance Care in Western Australia. Perth: St John Ambulance Service Australia, Western Australia Ambulance Service Inc.; 2005.

37. St John Ambulance Service Australia Northern Territory. Patient Management Procedures and Drug Protocols. Darwin: St John Ambulance Service Australia Northern Territory; 2005.

38. Tasmanian Ambulance Service. Tasmanian Protocol Index. Hobart: Tasmanian Ambulance Service; 2005.

39. Tippett V, Bonham R. Review of the evidence for prehospital administration ofbenzyl penicillin in meningococcal septicaemia - experience in Queensland. Journal of Emergency Primary Health Care 2005;3(1-2).

\section{Acknowledgment}

The author would like to thank Amee Morgans from the Monash University Centre for Ambulance and Paramedic studies for her helpful comments on earlier drafts of this paper.

\section{Author Disclosure}

The author has no financial, personal or honorary affiliations with any commercial organization directly involved or discussed in this study.

This Article was peer reviewed for the Journal of Emergency Primary Health Care Vol.4, Issue 1, 2006 